\title{
Investigation of the Surface Morphology and Structural Characterization of Palm Fiber Reinforced Acrylonitrile Butadiene Styrene (PF-ABS) Composites
}

\author{
Budrun Neher1,2*, Md. Abdul Gafur³, Muhammad Abdullah Al-Mansur4, \\ Md. Mahbubur Rahman Bhuiyan'², Md. Rakibul Qadir ${ }^{3}$, Farid Ahmed1 \\ ${ }^{1}$ Department of Physics, Jahangirnagar University, Dhaka, Bangladesh \\ ${ }^{2}$ Department of Physics, Comilla University, Comilla, Bangladesh \\ ${ }^{3}$ PP and PDC, Bangladesh Council of Scientific and Industrial Research, Dhaka, Bangladesh \\ ${ }^{4}$ BCSIR Laboratories, Dhaka, Bangladesh Council of Scientific and Industrial Research, Dhaka-1205, Bangladesh \\ Email: budrunneher@gmail.com
}

Received 2 March 2014; revised 6 April 2014; accepted 21 April 2014

Copyright (C) 2014 by authors and Scientific Research Publishing Inc.

This work is licensed under the Creative Commons Attribution International License (CC BY). http://creativecommons.org/licenses/by/4.0/

(c) (i) Open Access

\section{Abstract}

With the aid of Injection Moulding Machine (IMM) Palm fiber reinforced Acrylonitrile Butadiene Styrene (ABS) composites (PF-ABS) were prepared. Three sets of samples were prepared for three different wt\% (5\%, 10\% and $20 \%$ ) of fiber contents. Scanning Electron Microscopy (SEM), X-ray diffraction (XRD) and Fourier Transform Infrared Spectroscopy (FTIR) experiments were performed to study the surface morphology, microstructure (if it crystalline or noncrystalline) and new bond formation after preparation of the composites. SEM pattern shows that after addition of palm fiber in PF-ABS composites the brittleness increased due to creation of voids in the composites except $10 \%$ fiber content in PF-ABS. From XRD pattern it is clear that the palm fiber, ABS and PF-ABS composites are amorphous in nature. Moreover FTIR spectrum shows that there is no new bond formed after addition of palm fiber in ABS polymeric matrix to create PF-ABS composites.

\section{Keywords}

PF-ABS, IMM, Surface Morphology, Microstructure and Brittleness

\footnotetext{
"Corresponding author.
}

How to cite this paper: Neher, B., Gafur, Md.A., Al-Mansur, M.A., Rahman Bhuiyan, Md.M., Qadir, Md.R. and Ahmed, F. (2014) Investigation of the Surface Morphology and Structural Characterization of Palm Fiber Reinforced Acrylonitrile Butadiene Styrene (PF-ABS) Composites. Materials Sciences and Applications, 5, 378-386. 


\section{Introduction}

The use of natural plant fibres as reinforcement in polymer composites for making low cost engineering materials has generated much interest in recent years. New environmental legislation as well as consumer pressure has forced manufacturing industries (particularly automotive, construction and packaging) to search for new materials that can substitute for conventional non-renewable reinforcing materials such as glass fibre [1]. The natural fiber as reinforcement for composites has received increasing attention and polymer based composites having natural fiber as reinforcement have the potential to be attractive alternative to synthetic fiber composites. The advantages of natural plant fibres over traditional glass fibres are acceptable as good specific strengths and modulus, economical viability, low density, reduced tool wear, enhanced energy recovery, and reduced thermal and respiratory irritation and good biodegradability [2]. Natural plant fibre reinforced polymeric composite, also has some disadvantages such as the incompatibility between the hydrophilic natural fibres and hydrophobic thermoplastic and thermoset matrices requiring appropriate use of physical and chemical treatments to enhance the adhesion between fibre and the matrix [3].

Cellulosic fibers, like palm, henequen, sisal, coir, jute, bamboo, wood, paper in their natural condition, as well as, several waste cellulosic products such as shell flour, wood flour and pulp have been used as reinforcement agents of different thermosetting and thermoplastic resins [4]. Among these fibers, palm fiber is one of particular interest because palm fiber (Palmyra Palm) grows plenty in Bangladesh and all over the world. The properties of natural fibers largely depend on the chemical composition of the fiber. Palm fiber consists of $38.4 \%$ $\alpha$-cellulose, $30.9 \%$ hemicelullose, $27.3 \%$ lignin and 3\% - 5\% other chemicals on dry basis [5]. Composites made of palm fibers have moderate tensile and flexural properties compared with other natural fibers.

ABS is an engineering thermoplastic which consists of an amorphous, heterophasic polymer with very good mechanical properties, especially high impact resistance [6]. ABS is made by polymerizing styrene and acrylonitrile in the presence of polybutadiene. The proportions of contents in ABS can vary from $15 \%$ to $35 \%$ acrylonitrile, $5 \%$ to $30 \%$ butadiene and $40 \%$ to $60 \%$ styrene. The nitrile groups from neighboring chains, being polar, attract each other and bind the chains together, making ABS stronger than pure polystyrene. The styrene gives the plastic a shiny, impervious surface. The butadiene, a rubbery substance, provides resilience even at low temperatures [7]. These polymers are of low cost and provide excellent mechanical properties [8]. On the other hand, the ABS limitations are: poor flame and chemical resistance, and low thermal stability [9].

A large number of experiments have performed with natural fiber reinforced polymeric composites all over the world. Mohammad, N. N. B. et al. [10] carried out research on the morphological study of Kenaf fiber reinforced rPET/ABS composites. The distribution effects between $\mathrm{rPET} / \mathrm{ABS}$ and kenaf were studied through morphological characterization by using SEM. The result showed that there was increasing of the brittleness of composites when higher percentage of fiber was introduced and kenaf was fully interpenetrated into the composites. Seung-Hwan Lee et al. [11] showed that fibers were pulled out from the matrix in the fracture process, with large voids thereby being created. This findings suggest that the interaction between matrix and filler was very weak, resulting in less interfacial adhesion. A. H. Bhuiyan et al. [12] observed from the SEM taken on the fractured surface of the sample that the amount of voids increased with the increase of filler content, which tends to decrease tensile strength, elongation at break. Moreover small particles are seen to disperse inhomogeneously in the matrix of the composite. While the surface of the composites shows a few cracks, terraces and voids, a considerable amount of voids appear in the composites including different fracture processes in the neat iPP and the composites.

Palm fiber would be a good reinforcing agent in ABS and will be a good area of research. With a view to change the mechanical and physical properties of the composites, PF-ABS composites were prepared for different wt (\%) of PF in PF-ABS composites. Study of mechanical and physical properties of palm fiber reinforced ABS (PF-ABS) composites showed that tensile strength (TS) and flexural strength (FS) of the composites decreased with the addition of fiber content in the composites. On the other hand due to hydrophilic nature of the palm fiber, water absorption ability slightly increased with the increase of fiber content in the PF-ABS composites. Moreover water absorption ability became saturated after certain time [13]. In the present study the cause behind the decrement of TS and FS with the addition of fiber content in the PF-ABS composites would be carried out by investigating surface morphology using SEM and characterization of the structure using XRD and FTIR spectroscopy. 


\section{Materials and Methods}

\subsection{Sample Collection and Composite Preparation}

Palm leaves of ten different aged trees (five of them are above 10 years and another five of them are below 10 years) were collected from Burura region of Comilla district. Dividing ends of the middle hard part of the leaves were hammered. Hard part of the leaves was immersed in water for 20 days to rotten. Rotten materials were cleaned and fiber were then separated, dried under sun light and kept at $100^{\circ} \mathrm{C}$ for $24 \mathrm{hrs}$ for partial removal of moisture. ABS was collected from local market of old Dhaka of Bangladesh. Palm fiber was cut to $1-2 \mathrm{~mm}$ in sized. ABS and small palm fiber were dried in dryer at $50^{\circ} \mathrm{C}$ for $24 \mathrm{hrs}$. Fined palm fiber and ABS polymer put into the injection moulding machine. This mixture was heated at $150^{\circ} \mathrm{C}$ inside the injection molded machine and molten mixture become composite and come out of the IMM. This composite was poured into different shape of die for different test. Three sets of composites samples (with 5\%, $10 \%$ and $20 \%$ fiber content) were prepared to carry out this research.

\subsection{Characterization of PF-ABS Composite}

\subsubsection{Surface Morphology of PF-ABS Composite}

To perform SEM, each sample was cut from the end of fracture surfaces with an appropriate size. The sample surface of the composites was coated with gold in agar auto sputter coater (Model 108A, England) before subjected to scanning electron microscope. The fiber matrix adhesion of the fracture surface of the composites was examined by Scanning Electron Microscope (Model: JEOL.JSM-6490 LA Analytical scanning electron microscope) [14] at a magnification of $\times 100, \times 500$ and $\times 1000$ with a maximum operating voltage of $20 \mathrm{kV}$.

\subsubsection{Structural Characterization of PF-ABS Composite}

1) X-ray diffraction (XRD) analysis of PF-ABS composite

XRD studies of the neat ABS sample and PF-ABS composites for different wt (\%) were performed by an X-ray diffractometer (Model: Bruker AXS D8 ADVANCE) using $C u K_{\alpha}$-radiataion of wavelength, $\lambda=1.5418 \AA$.

\section{2) Fourier Transform Infrared Spectroscopy (FTIR)}

FTIR spectra of the samples were recorded at room temperature by using a double beam IR spectrometer (SHIMADZU, FTIR-8900 spectrometer, Japan) in the wave number range of $625-4000 \mathrm{~cm}^{-1}$. In this experiment, the solid samples were finely pulverized with pure, dry $\mathrm{KBr}$, the mixture is pressed in a hydraulic press to form a transparent pellet.

When the beam of light passes through the sample, it becomes less intense due to the absorption of certain frequencies. The absorbance of the sample at a particular frequency can be calculated as

$$
\begin{array}{r}
A=\log \left(\frac{\mathrm{I}_{0}}{\mathrm{I}}\right) \\
\text { Transmittance, } \mathrm{T}=\frac{\mathrm{I}}{\mathrm{I}_{\mathrm{O}}}
\end{array}
$$

where $\mathrm{I}_{\mathrm{o}}$ and $\mathrm{I}$ are the intensity of beam before and after interaction. The FTIR spectrum of the sample pellet is recorded in transmittance (\%) mode.

\section{Result and Discussions}

\subsection{Surface Morphology of PF-ABS Composite}

The morphological study of the PF-ABS composites carried out by SEM to determine the adhesion between fiber and ABS polymer, the reason of the mode or cause of failure and to correlate to the mechanical data. Scanning electron micrograph for different wt (\%) of fiber in PF-ABS composites are shown in Figures 1-4 at a magnification of $\times 100, \times 500$ and $\times 1000$ with a maximum operating voltage of $20 \mathrm{kV}$.

Figure 1 represents the surface of ABS is smooth. When the magnification increased to $\times 500 \& \times 1000$ times, few circular voids are observed (showing by red arrow) which imply that low adhesion of ABS polymeric material in IMM. 


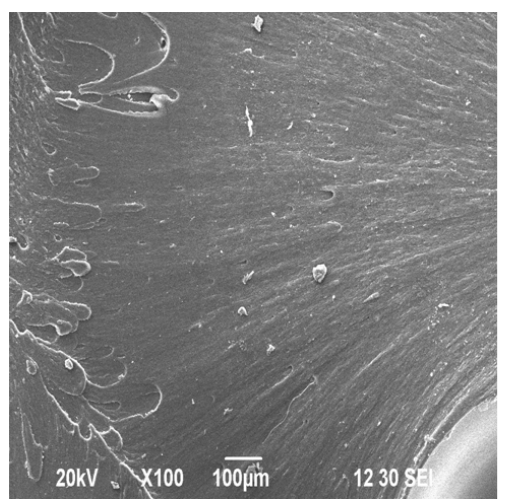

(a)

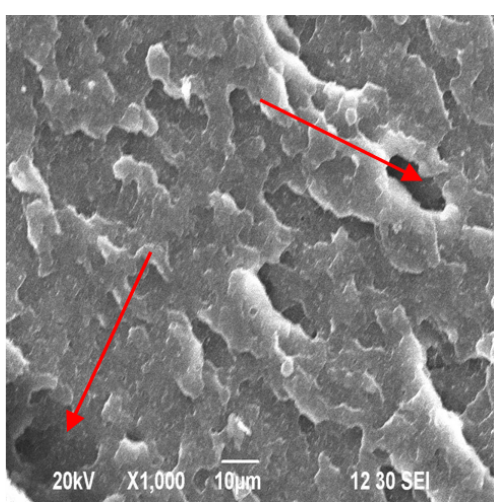

(b)

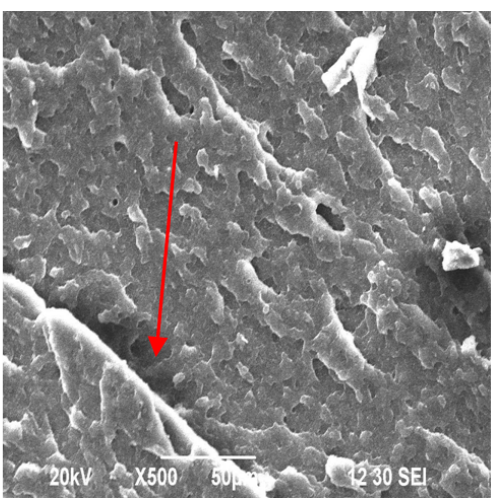

(c)

Figure 1. SEM Micrograph of ABS Polymer for different magnification: (a) $\times 100$ (b) $\times 500$ and (c) $\times 1000$ at operating voltage of $20 \mathrm{kV}$.

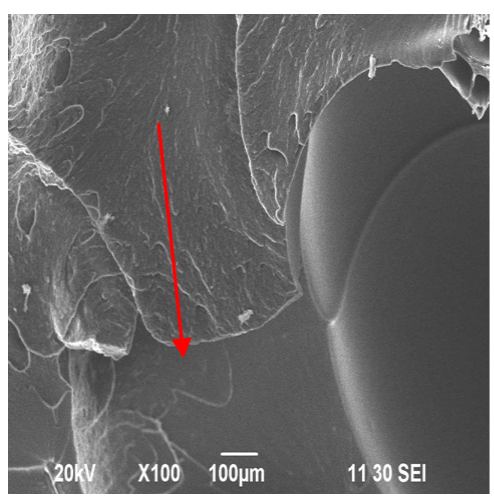

(a)

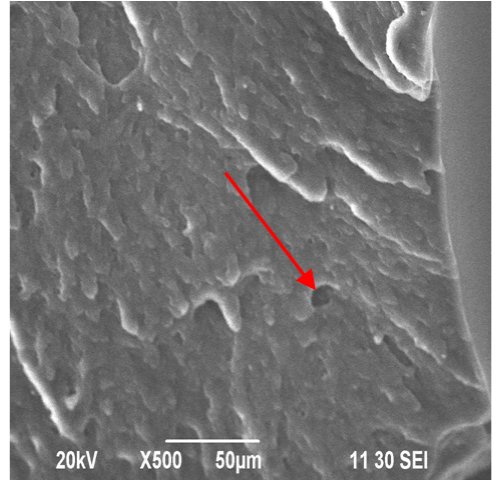

(b)

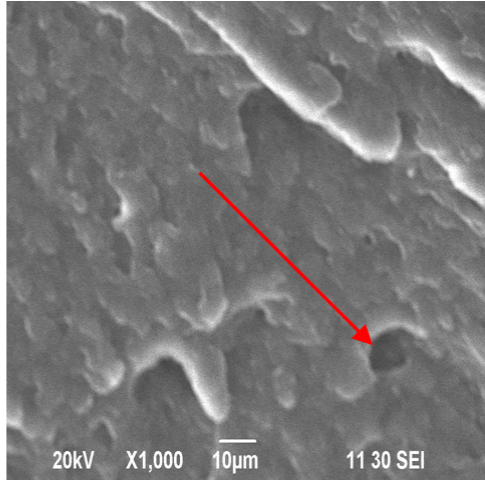

(c)

Figure 2. SEM Micrograph of PF-ABS composite with 5\% fiber for different magnification: (a) $\times 100$ (b) $\times 500$ and (c) $\times 1000$ at operating voltage of $20 \mathrm{kV}$.

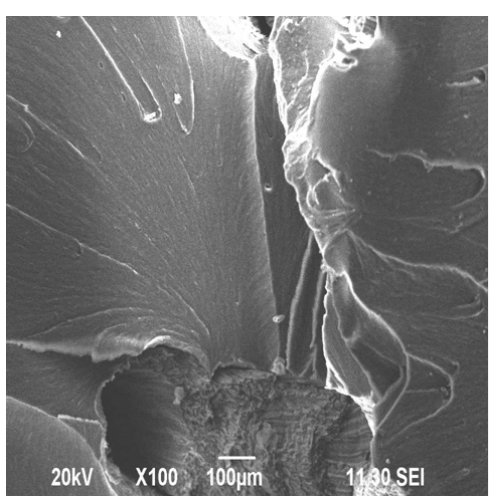

(a)

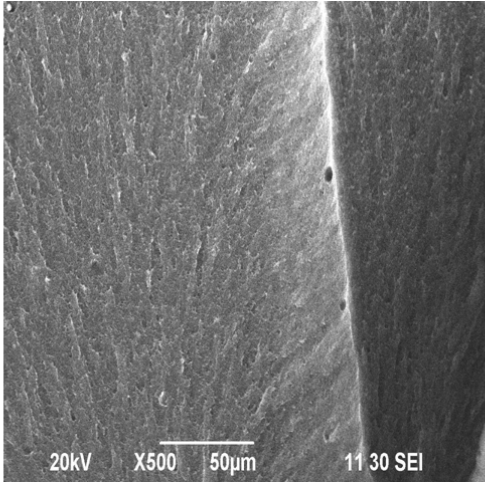

(b)

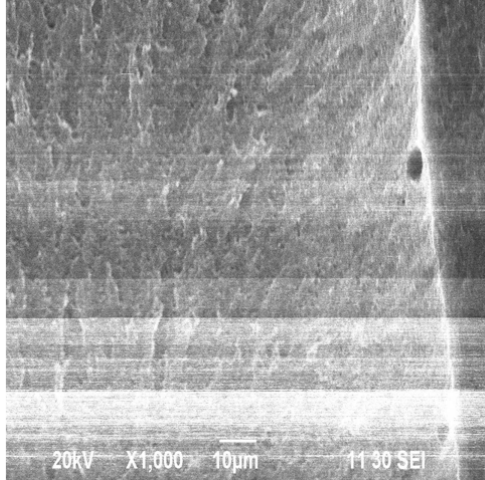

(c)

Figure 3. SEM Micrograph of PF-ABS composite with $10 \%$ fiber for different magnification: (a) $\times 100$ (b) $\times 500$ and (c) $\times 1000$ at operating voltage of $20 \mathrm{kV}$.

The scanning electron micrograph of composite with 5\% fiber shows (in Figure 2) that the composite surface is rough. When the magnification increased to $\times 500$ and $\times 1000$ times, number of circular voids increased. This may because the fibers may pull out when bending or fiber may contain little amount moisture content or air may be passed when palm fiber mixing with ABS in IMM. When heated in injection moulding machine, moisture content vaporized and create voids. 


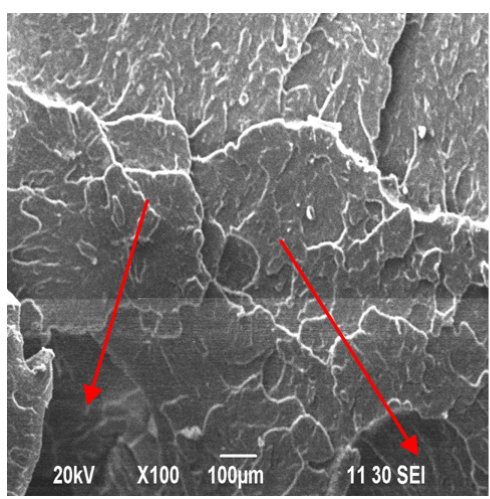

(a)

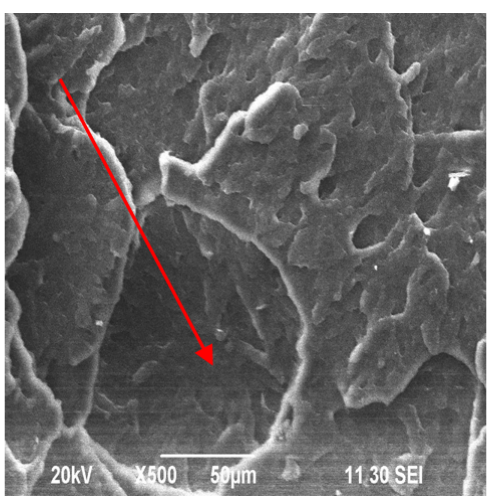

(b)

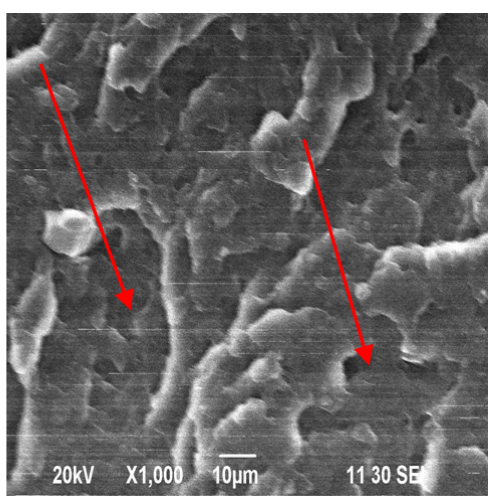

(c)

Figure 4. SEM Micrograph of PF-ABS composite with $20 \%$ fiber for different magnification: (a) $\times 100$ (b) $\times 500$ and (c) $\times 1000$ at operating voltage of $20 \mathrm{kV}$.

Figure 3 provides that the scanning electron micrograph with $10 \%$ fiber in PF-ABS. It represents that the composite surface is comparatively smooth than the composite with $5 \%$ fiber. It also shows that fiber breakdown and it tightly attached to the polymer matrix, which means that comparatively strong adhesion between fiber and polymer matrix. This may slightly increased the strength of the composites. The area of the circular voids is comparatively small and surface become comparatively smooth. Moreover it is shown that the interfacial adhesions between fiber-matrix remain in good performance in $10 \%$ fiber in PF-ABS. The scanning electron micrograph of PF-ABS composite (shown in Figure 4) with 20\% fiber shows that the composite surface is rougher. With the increase of fiber contents in the polymeric matrix, the number of voids increased, area of each void also increased. This means low adhesion between fiber and polymeric matrix, tends to decrease the strength of the composites.

The natural fiber has poor wettability, incompatibility with some polymeric matrices and high moisture absorption [15]. Due to high moisture absorption properties, there are formations of void in the composites which can reduce the mechanical properties of composite such as tensile and flexural strength [10]. With the increase of the fiber content in the PF-ABS composites, the number of void in the composite increased which makes the composites brittle. Brittle materials tend to show very low elongation because they do not plastically deformed. Increasing of the brittleness of samples is due to the formation of the void in the composite [10]. Moreover obvious cracked section propagates at composites surface, which indicates weak interfacial area between fiber-matrix [16]. Finally it can be obvious that the tensile strength (TS), flexural strength (FS), hardness of the composites decreased with the increase of fiber content except $10 \%$ fiber content due to the formation of voids, fiber pullout and the propagation of some crack section over the composites [11] [12] [17].

\subsection{Structural Characteristics of PF-ABS Composite}

\subsubsection{X-Ray Diffraction (XRD) Analysis of PF-ABS Composite}

In XRD, the intensity of every component's pattern is proportional to the amount of the component present [18].

Figure 5 and Figure 6 show the XRD pattern of palm fiber, ABS polymer and PF-ABS composite having 5\%, $10 \%$ and $20 \%$ fiber. It is seen from XRD spectrum of palm fiber, ABS polymer and PF-ABS composite, all of them give broad peaks, which imply that the PF-ABS composite including ABS polymer are amorphous in nature. The X-ray diffracted from ABS polymer resin in all cases is same also for fiber they diffracted for all sample in similar way. For this reason all spectra are more or less similar for the samples. There is no sharp peak which reveals non crystalline nature of the composites.

\subsubsection{Fourier Transform Infrared (FTIR) Spectroscopy}

Figures 7-9 presents the FTIR spectrum in the frequency range $\left(625-4000 \mathrm{~cm}^{-1}\right)$ for palm fiber, ABS polymer and PF-ABS composites respectively.

The major peak at $669,720,986,1038,1138,1558 \mathrm{~cm}^{-1}$ are due to the presence of $\mathrm{C}=\mathrm{C}$ out of plane bending, $\mathrm{C}-\mathrm{H}$ bending, $=\mathrm{C}-\mathrm{H}$ out of plane bending, $\mathrm{C}-\mathrm{H}$ in plane bending, $\mathrm{C}-\mathrm{C}$ skeletal and $\mathrm{C}=\mathrm{O}$ bending bonds in the 


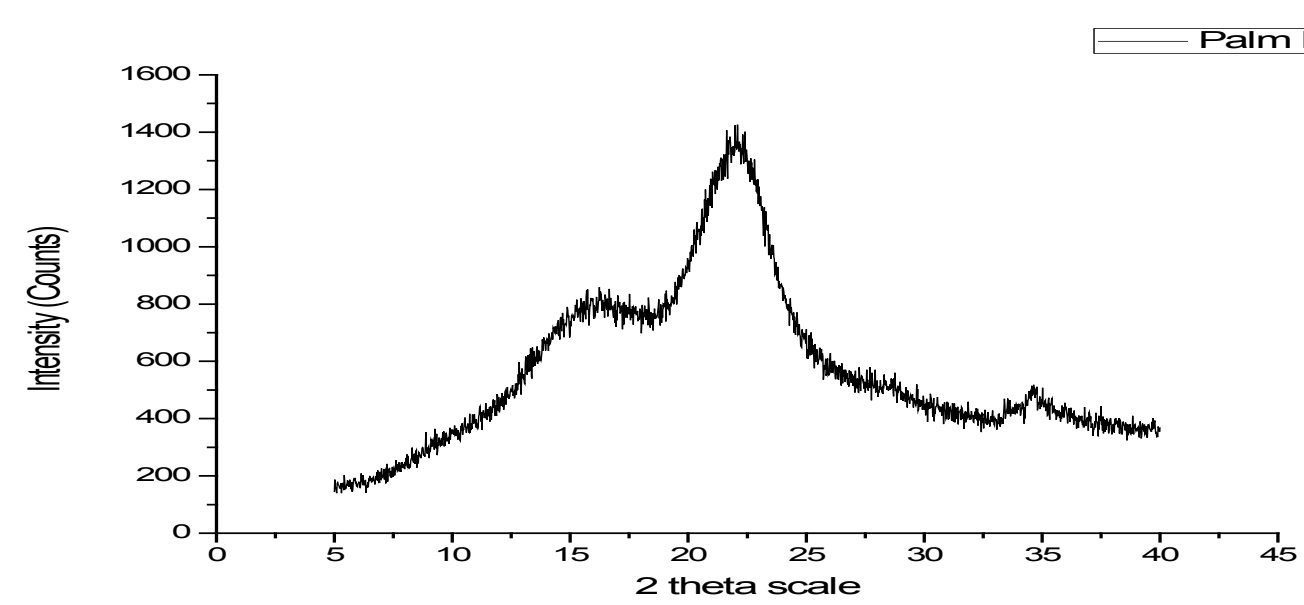

Figure 5. XRD pattern of Palm fiber.

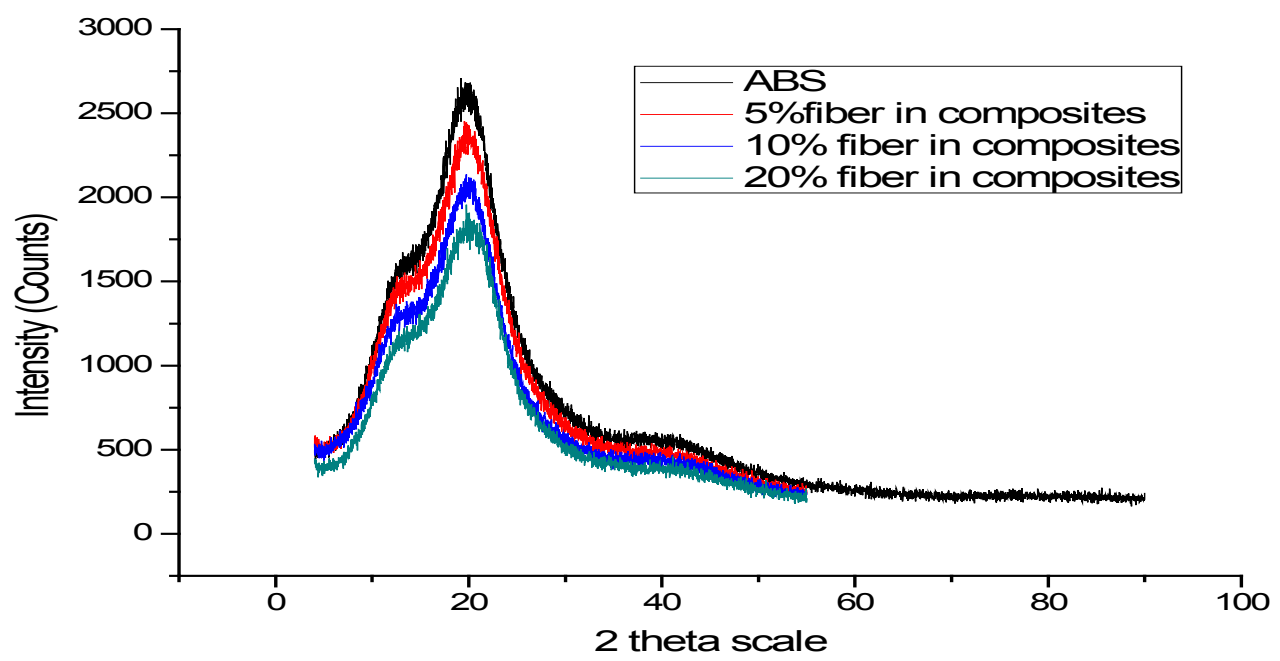

Figure 6. XRD pattern of ABS polymer and PF-ABS composites for different wt (\%) of PF fiber.

palm fiber. The major peaks for ABS polymer at about 698, 758, $910-964,1028,1452-1493,2250,2918 \mathrm{~cm}^{-1}$ are due to the presence of $\mathrm{C}-\mathrm{H}$ out of plane, $=\mathrm{C}-\mathrm{H}$ out of plane bending, $\mathrm{C}-\mathrm{H}$ bending, $\mathrm{C}-\mathrm{H}$ in plane bending, asymmetry $\mathrm{C}-\mathrm{H}$ bending, $\mathrm{C} \equiv \mathrm{N}$ stretching and symmetric $\mathrm{C}-\mathrm{H}$ stretching bonds. After formation of $\mathrm{PF}-\mathrm{ABS}$ composites 667, 758, 910 - 966, 1028, 1155 - 1180, 1219 - 1240, 1317 - 1396, 1454 - 1495, 1506 - 1558,1601 1636,2240 and $2920 \mathrm{~cm}^{-1}$ peaks are found due to the $\mathrm{C}-\mathrm{H}$ out of plane, $=\mathrm{C}-\mathrm{H}$ out of plane bending, $\mathrm{C}-\mathrm{H}$ bending, $\mathrm{C}-\mathrm{H}$ in plane bending, $\mathrm{C}-\mathrm{C}$ skeletal, $\mathrm{C}-\mathrm{H}$ twisting, symmetry $\mathrm{C}-\mathrm{H}$ bending, asymmetry $\mathrm{C}-\mathrm{H}$ bending, $\mathrm{C}=\mathrm{O}$ bending, $\mathrm{C}=\mathrm{C}$ stretching, $\mathrm{C} \equiv \mathrm{N}$ stretching and symmetry $\mathrm{C}-\mathrm{H}$ stretching bonds. After comparison among spectrum of the PF, ABS and PF-ABS composites, it reveals that no new bonds are formed due to mixture of palm fiber with ABS in IMM.

\section{Conclusion}

The scanning electron microscopy (SEM), X-ray diffraction (XRD) and Fourier Transform Infrared Spectroscopy (FTIR) experiments were performed to investigate the decrement nature of mechanical property like tensile strength, flexural strength and hardness due to addition of palm fiber in PF-ABS composites. The scanning elec- 


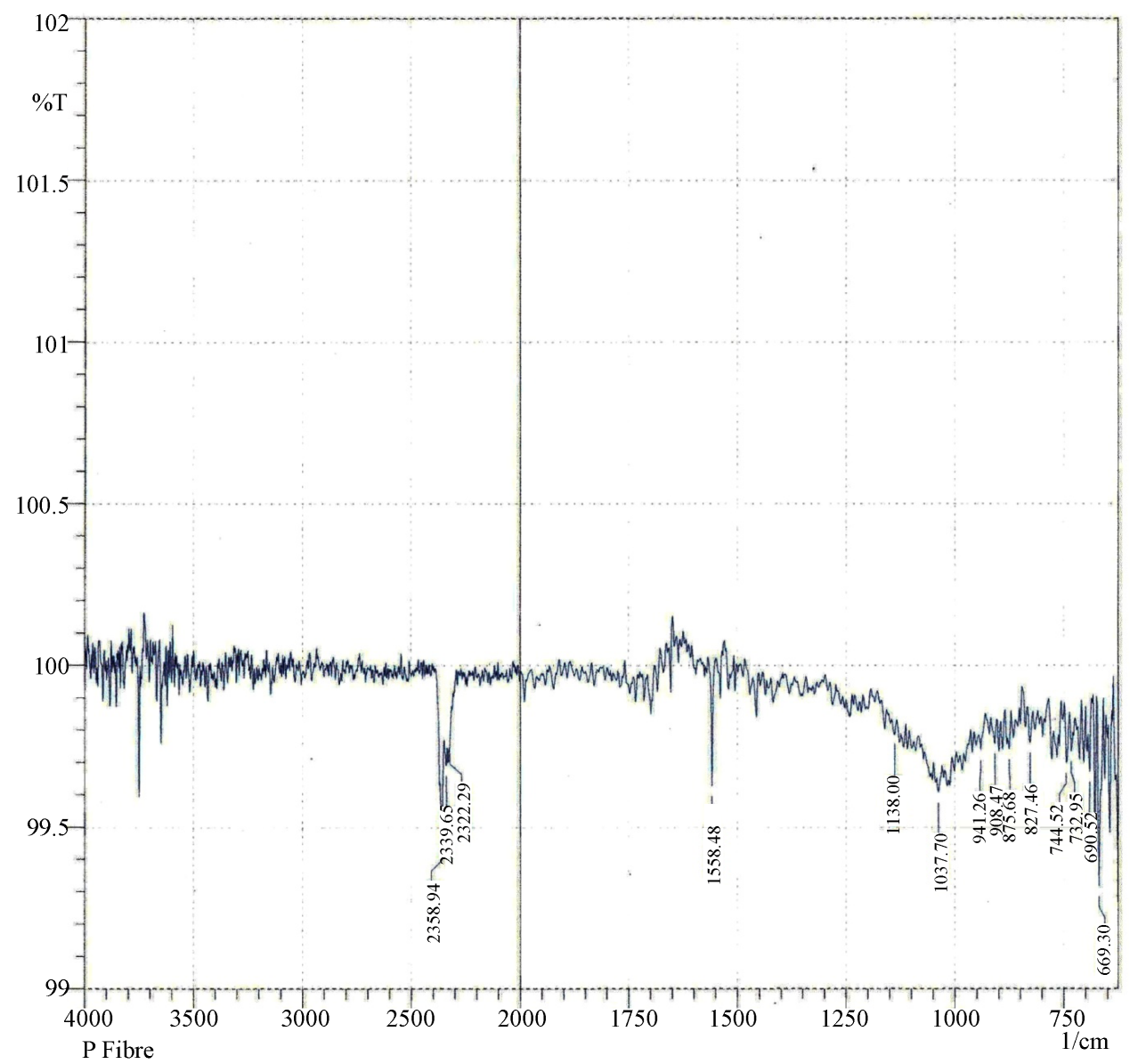

Figure 7. FTIR spectrum of palm fiber.

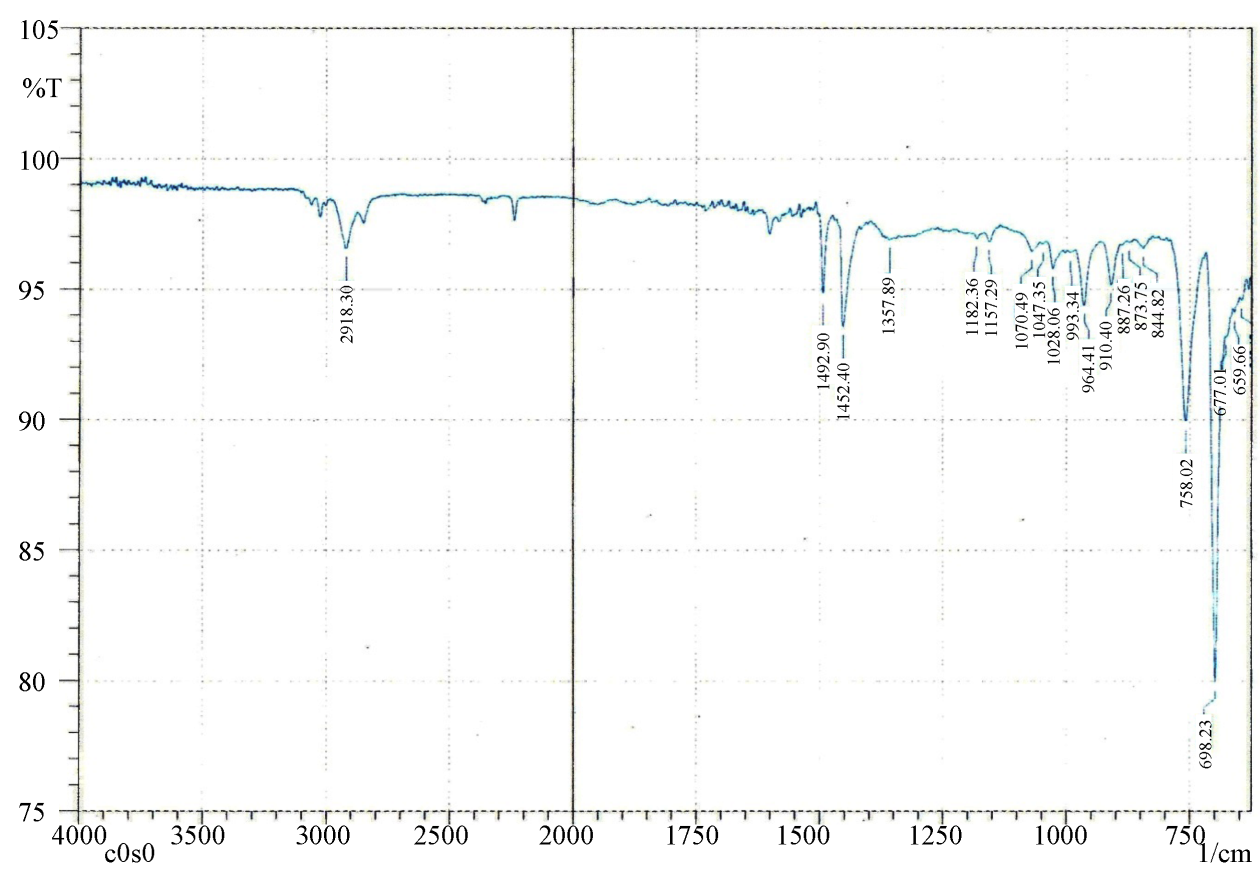

Figure 8. FTIR spectrum of raw ABS polymer. 


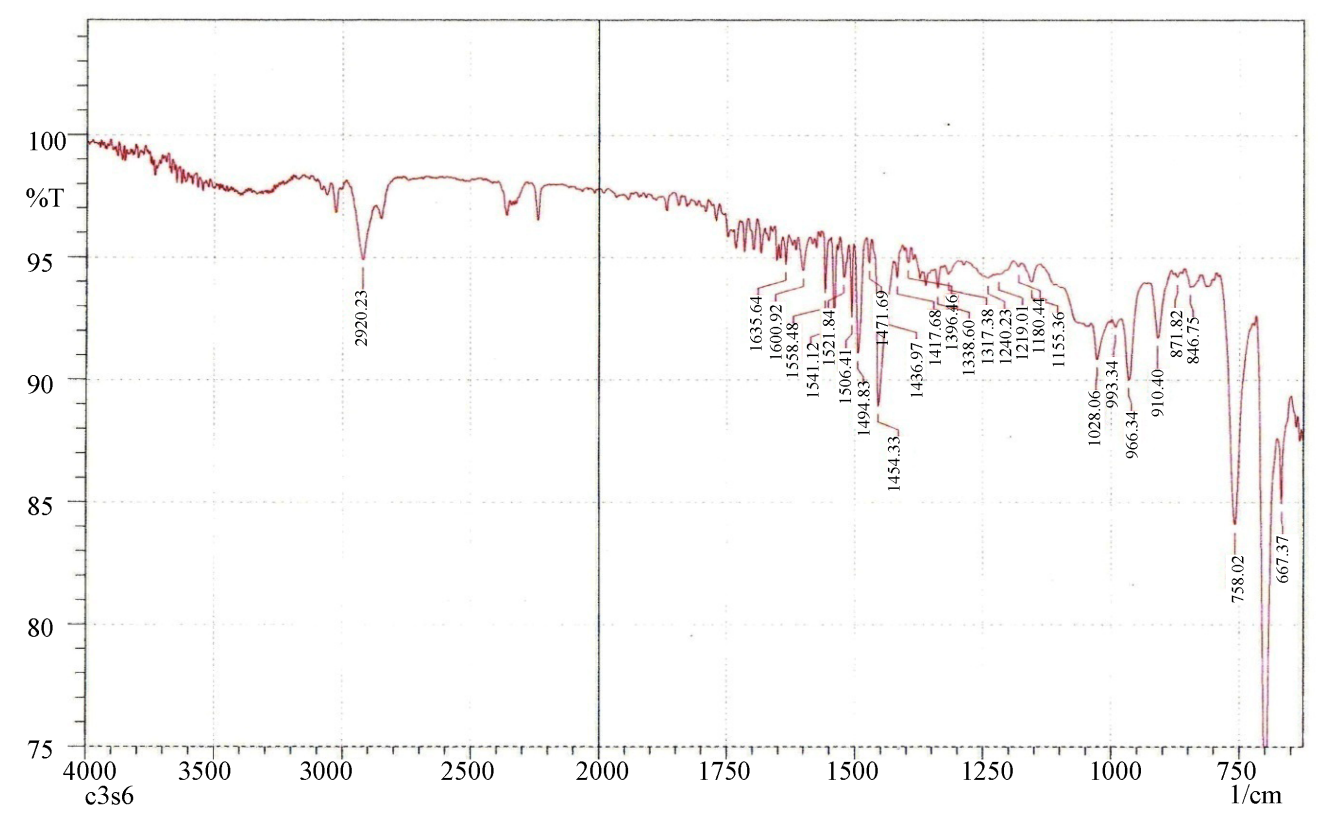

Figure 9. FTIR spectrum of PF-ABS composite.

tron micrograph shows that with the addition of $\mathrm{PF}$ in $\mathrm{ABS}$ composites, the number of voids increased except $10 \%$ fiber content in PF-ABS. The area of voids also increased. Some cracks are found in SEM micrograph which reveals brittleness of composites. Fiber pulled out for 5\% and 20\% PF-ABS composites but for $10 \%$ fiber attached with composites due to better adhesion between PF-ABS composites. The XRD pattern and FTIR spectrum show that the composites are amorphous in nature and no new bond was formed.

\section{References}

[1] Dhakal, H.N., Zhang, Z.Y. and Richardson, M.O.W. (2007) Effect of Water Absorption on the Mechanical Properties of Hemp Fibre Reinforced Unsaturated Polyester Composites. Composites Science and Technology, 67, 1674-1683. http://dx.doi.org/10.1016/j.compscitech.2006.06.019

[2] Bolton, J. (1995) The Potential of Plant Fibres as Crops for Industrial Use. Outlook Agric, 24, 85-89.

[3] Gassan, J. and Cutowski, V.S. (2000) Effect of Corona Discharge and UV Treatment on the Properties of Jute-Fiber Epoxy Composites. Composites Science and Technology, 60, 2857-2863. http://dx.doi.org/10.1016/S0266-3538(00)00168-8

[4] Herrera-Franco, P.J. and Valadez-Gonzalez, A. (2005) A Study of the Mechanical Properties of Short Natural-Fiber Reinforced Composites. Composites: Part B, 36, 597-608. http://dx.doi.org/10.1016/S0266-3538(00)00168-8

[5] Maniruzzaman, M., Rahman, M.H. and Akther Zaman, M.A. (2005) Composition of Agave Atroverance Fiber. Jahangirnagar University Journal of Science, 28, 23-30.

[6] Salari, D. and Ranjbar, H. (2008) Study on the Recycling of ABS Resins: Simulation of Reprocessing and Thermo-oxidation. Iranian Polymer Journal, 17, 599-610.

[7] Acrylonitrile Butadiene Styrene (ABS) (2010) http://en.wikipedia.org/wiki/Acrylonitrile butadiene styrene

[8] Reena, G., Sangita and Verinder, K. (2011) FT-IR Studies of E-Plastic Obtained from Obsolete Computers. Journal of Chemical and Pharmaceutical Research, 3, 660-667.

[9] Krache, R. and Debbah, I. (2011) Some Mechanical and Thermal Properties of PC/ABS Blends. Materials Sciences and Applications, 2, 404-410. http://dx.doi.org/10.4236/msa.2011.25052

[10] Mohammad, N.N.B. and Arsad, A. (2013) Mechanical, Thermal and Morphological Study of Kenaf Fiber Reinforced rPET/ABS Composites. Malaysian Polymer Journal, 8, 8-13.

[11] Lee, S.H. and Wang, S. (2006) Biodegradable Polymers/Bamboo Fiber Biocomposites with Bio-Based Coupling Agent. Composites: Part A, 37, 80-91. http://dx.doi.org/10.1016/j.compositesa.2005.04.015

[12] Bhuiyan, A.H., Mina, M.F., Seema, S., Khan, M.M., Rahman, M.J. and Gafur, M.A. (2011) Structural, Elastic and 
Thermal Properties of Titanium Dioxide Filled Isostatic Polypropylene. Journal of Polymer Research, 18, 1073-1079. http://dx.doi.org/10.1007/s10965-010-9509-y

[13] Neher, B., Bhuiyan, M.M.R., Kabir, H., Qadir, M.R., Gafur, M.A. and Ahmed, F. (2014) Study of Mechanical and Physical Properties of Palm Fiber Reinforced Acrylonitrile Butadiene Composite. Materials Sciences and Applications, 5, 39-45.

[14] Khan M.A., Islam, T., Arifur Rahman, M., Islam, J.M.M., Khan, R.A., Gafur, M.A., Mollah, M.Z. I. and Alam, A.K.M. (2010) Thermal, Mechanical and Morphological Characterization of Jute/Gelatin Composites. Polymer-Plastics Technology and Engineering, 49, 742-747. http://dx.doi.org/10.1080/03602551003652698

[15] Taj, S., Murawar, M.A. and Khan, S. (2007) Natural Fiber-Reinforced Polymer Composites. Proceedings of Pakistan Academy of Sciences, 44, 129-144.

[16] Tan, C., Ahmad, I. and Heng, M. (2001) Characterization of Polyester Composites from Recycled Polyethylene Terephthalate Reinforced with Empty Fruit Bunch Fibers. Materials and Design, 32, 4493-4501. http://dx.doi.org/10.1016/j.matdes.2011.03.037

[17] Moniruzzaman, M., Moniruzzaman, M., Gafur, M.A. and Santulli, C. (2009) Lady's Finger Fibres for Possible Use as a Reinforcement in Composite Materials. Journal of Biobased Materials and Bioenergy, 3, 1-5.

[18] Budnikov, P.P. (1964) The Technology of Ceramic and Refractories. M. I. T. Press, Cambridge. 